\title{
Avaliação da escalade design da simulação em acadêmicos de medicina
}

\author{
Evaluation of the simulation design \\ scale in medical students
}

\section{Marina Pereira Quadros ${ }^{1}$ (1) Maristela Rodrigues Sestelo ${ }^{2}$ (1) lêda Maria Barbosa Aleluia 3 (1)}

\begin{abstract}
1Autora para correspondência. Escola Bahiana de Medicina e Saúde Pública (Salvador). Bahia, Brasil. marinaquadros16.2@bahiana.edu.br
\end{abstract}
2-3Escola Bahiana de Medicina e Saúde Pública (Salvador). Bahia, Brasil. maristelasestelo@bahiana.edu.br, iedaleluia@bahiana.edu.br

RESUMO | INTRODUÇÃO: A simulação realística tem sido um método de aprendizagem amplamente utilizado no Brasil, sobretudo no ensino médico. Para reproduzir os cenários de atuação médica de forma mais fidedigna, o Design da simulação precisa ser bem planejado para levar o aluno ao maior nível de aproveitamento possível. OBJETIVOS: Descrever a avaliação da qualidade das características do desenho do cenário da simulação pelos dos alunos dos cursos de medicina. MÉTODOS: Estudo observacional, descritivo, de corte transversal realizado com estudantes do curso de medicina, no período de fevereiro de 2019. A amostra final foi de 90 estudantes. A Escala de Design da Simulação foi utilizada para a avaliação da estrutura do cenário da simulação. A partir da escala, as avaliações do Design da simulação pelos alunos foram tabuladas e comparadas através das médias e desvio padrão. RESULTADOS/DISCUssão: Todos os itens analisados da Escala de Design da Simulação obtiveram resultados positivos na avaliação, assim como a importância da elaboração adequada do Design. Aquele que obteve concordância predominante foi o domínio "Realismo" com média de 4,94 e desvio padrão de 0,20 e importância predominante foi o domínio “Feedback/ reflexão", com média de 4,95 e desvio padrão de 0,16. CONCLUSÃo: Os resultados deste estudo indicam percepções predominantemente positivas dos alunos do curso de medicina acerca do design construído para a simulação aplicada e da sua importância para o processo e aprendizagem. Além disso, pode-se de apontar os aspectos que precisam de melhor desenvolvimento e aprimoramento da simulação, assim como ressaltar os pontos positivos de destaque.

PALAVRAS-CHAVE: Simulação. Simulação realística. Ensino médico. Aprendizado.
ABSTRACT | BACKGROUND: The High-Fidelity Simulation has been a learning method widely used in Brazil, especially in medical education. In order to reproduce the scenarios of medical care more efficiently and reliably, the Design of Simulation needs to be well planned to take the student to the highest possible level of achievement. AIMS: Measure the evaluation of the quality of the characteristics of the design of the simulation scenario by the students of medical courses. METHODS: Observational, descriptive, cross-sectional study carried out with medical students in February 2019. The final sample was 90 students. The Likert Simulation Design Scale was used to assess the structure of the simulation scenario; the data were tabulated and compared using means and standard deviation. RESULTS/ DISCUSSION: Given the analyzed items of the Simulation Design Scale, all obtained positive results in the evaluation and the importance of proper design elaboration. The one that obtained predominant agreement was "Realism," with an average of 4,94 and a standard of 0,20 . The one that obtained predominant importance was the factor "Feedback/reflection," with an average of 4,95 and a standard of 0,16 . CONCLUSION: The results of this study indicate predominantly positive perceptions of medical students about the design built for applied simulation and its importance for the process and learning. In addition, it is possible to point out the aspects that need better development and improvement of the simulation and highlight the positive points of prominence.

KEYWORDS: Simulation. High-Fidelity simulation. Realistic simulation. Medical education. Learning. 


\section{Introdução}

Os atuais métodos de ensino em saúde no Brasil estão sendo modificados de acordo a necessidade vigente da diminuição de possíveis erros na atuação do profissional. Nessa perspectiva, tem-se percebido nos cursos da área de saúde uma crescente tendência em implementar de atividades que valorizem o uso dos saberes cognitivos associados a experiências emocionais como forma de maior fixação do conteúdo adquirido. ${ }^{1,2}$ Essa nova perspectiva envolve o desenvolvimento e aprimoramento de habilidades de liderança, trabalho em equipe e raciocínio clínico que serão necessárias quando o aluno estiver frente a uma situação de atendimento médico real. $\underline{3}$

A simulação realística é um método inovador que consegue anexar ao conhecimento teórico, vínculos emocionais construídos a partir de experiências semelhantes a situações reais. ${ }^{4}$ Apesar de recente no ensino em saúde do Brasil, nos Estados Unidos e Europa, a simulação realística já se tornou um instrumento de ensino fortemente consolidado. ${ }^{3}$

Nesse contexto, a proposta do método é que o aluno possa vivenciar uma situação prática semelhante à realidade, porém desprovida de risco ao paciente e a si mesmo. Portanto, a participação e atuação em cenários clínicos reconstruídos oferecem ao aluno uma oportunidade de demonstrar e adquirir habilidades, tornando o processo de aprendizado mais prazeroso e estimulante ao aluno.2,3

Para que o aluno tenha uma experiência completa e um alto aproveitamento do método, o Design da simulação deve se aproximar ao máximo de uma situação real. Nesse cenário, a simulação deve ser programada para incluir situações, fatores imprevistos ao aluno e variáveis que ocorrem também na vida real.

Partindo de um cenário criado, possuindo características de alta semelhança a situações reais, o aluno participa das simulações, promove maior segurança ao paciente, bem como melhoria da equipe, vivencia experiências ativas através das simulações e tem a oportunidade de aprender através das reflexões baseadas nos erros e acertos cometidos. ${ }^{5}$

Através da simulação realística, o aprendizado do aluno é facilitado de modo que as complicações decorrentes de tratamentos inadequados sejam minimizadas, aumentando a segurança aos pacientes. Além do conhecimento de abordagens técnicas, o aluno pode desenvolver habilidades sobre como gerenciar o atendimento em situações complicadas, de estresse, trabalho de equipe e como se relacionar adequadamente com pacientes e seus familiares. $\underline{5}$

O cenário da simulação é uma ferramenta crucial para a que o aluno tenha o melhor aproveitamento do método de ensino. Tendo essa preocupação em vista, recomenda-se que a estratégia da construção do cenário siga um Design que contenha os objetivos devidamente esclarecidos, fidelidade e aproximação do cenário à realidade, capacidade de resolução de problemas correspondentes ao estágio da graduação do aluno, apoio ao estudante durante a atividade e o momento para debriefing/feedback. Diante disso, para avaliar a qualidade dos cenários desenvolvidos nos cursos de enfermagem, a National League for Nursing criou a The Simulation Design Scale (student version), que funciona como um método voltado ao aprimoramento do método de ensino. $\frac{6}{-}$

A The Simulation Design Scale (student version) já foi utilizada em outros cenários clínicos com profissionais de saúde da área de enfermagem. Estudos prévios ${ }^{6,7}$ demonstram boa confiabilidade no método e, por isso, pode-se ter uma boa perspectiva na aplicação da escala também em atividades de simulação dos demais cursos da área de saúde.

O presente estudo tem o objetivo descrever a avaliação da qualidade das características do desenho do cenário da simulação pelos alunos do curso de medicina, com o propósito de contribuir com a literatura nacional sobre o tema em questão, compreendendo profissionais multidisciplinares da área de saúde. 


\section{Metodologia}

Trata-se de um estudo de caráter observacional, descritivo, de corte transversal, que foi realizado com estudantes do curso de medicina do $5^{\circ}$ semestre no período de fevereiro de 2019. A atividade, inserida no componente curricular de Semiologia, se iniciou com instruções gerais sobre as etapas da simulação. Em seguida, foi exposto ao aluno que se voluntariou a participar da atividade a queixa principal que a paciente traria naquele cenário e foi orientado que o mesmo se comportasse e agisse da mesma forma que faria caso estivesse atendendo um paciente real em ambiente ambulatorial. O aluno que protagonizou a simulação, então, realizou o atendimento, enquanto os outros colegas da turma o assistiam. Em um terceiro momento, foi realizado uma etapa de feedback, que tinha o objetivo de enaltecer os pontos positivos executados pelo aluno voluntário, pontuar também pontos de melhoria e provocar reflexões sobre o cenário, tendo em vista as diferentes perspectivas de todos os alunos que estavam ali presentes na atividade. Após completar todas as etapas da atividade da simulação (preparação, simulação e feedback), cada aluno respondeu uma única vez a Escala de Design da Simulação (versão do aluno), na versão validada para a língua portuguesa, um questionário que corresponde a avaliação das características do desenho do cenário da simulação. O questionário era de caráter autoaplicável, sem tempo limite para ser respondido, que consiste na avaliação de cinco domínios: objetivos e informações da simulação, do apoio, da resolução de problemas, do feedback/reflexão e do realismo. Todos esses aspectos, juntos, somavam 20 itens a serem avaliados através do grau de concordância com a assertiva e grau de importância daquilo no cenário da simulação. Em ambas as subescalas, de concordância e importância, a pontuação para cada item poderia variar de 1 a 5, correspondendo respectivamente: 1 - Discordo totalmente da afirmação; 2 - Discordo da afirmação; 3 - Indeciso; 4 - Concordo com a afirmação; 5 - Concordo totalmente com a afirmação; NA - Não se aplica; e 1 - Não é importante; 2 - Pouco importante; 3 - Neutro; 4 - Importante; 5 - Muito Importante.
Os dados foram analisados através da plataforma IBM SPSS Statistics 22, onde foram calculados a média e o desvio padrão do desempenho de cada item e domínio. Foram analisados os dados de todos os alunos dos cursos de medicina que estiveram presentes na atividade da simulação com tema "Demência", que responderam o questionário. Foram excluídos, para a composição da amostra final, os alunos que apresentaram questionários mal preenchidos ou incompletos. Foram coletados um total de 110 questionários. Houve dispensa do TCLE para o estudo pelo comitê de ética em pesquisa. Dentre esses, foram utilizados os dados presentes em 90 questionários que foram selecionados de acordo aos critérios de inclusão e exclusão.

Foram construídas tabelas para exposição dos resultados, os quais também serão discutidos com o embasamento teórico disponível na literatura até o presente momento.

O presente projeto de pesquisa foi submetido e aprovado pelo Comitê de Ética em Pesquisa da Escola Bahiana de Medicina e Saúde Pública, com o CAAE número 19550619.3.0000.5544.

\section{Resultados}

Os resultados encontrados a partir da análise dos 90 questionários válidos foram subdivididos de acordo às categorias de avaliação das afirmações sobre o Design da simulação. 
A tabela 1 corresponde aos resultados da análise das médias e desvio padrão dos itens numerados de 1 a 5 que correspondem a afirmações sobre "Objetivos e Informações" da simulação.

Tabela 1. Médias, número de respostas válidas e desvio padrão relativos ao domínio "Objetivos e Informações"

\begin{tabular}{l|c|c|c|c|c}
\hline \multicolumn{1}{c|}{ Comando } & Média de concordância & Desvio padrão & $\begin{array}{c}\text { Desvio padrão Número } \\
\text { de respostas válidas }\end{array}$ & $\begin{array}{c}\text { Média de } \\
\text { importância }\end{array}$ & 4,91 \\
respostas válidas
\end{tabular}


A tabela 2 corresponde aos resultados da análise das médias e desvio padrão dos itens numerados de 6 a 9 que correspondem a afirmações sobre "Apoio" da simulação.

Tabela 2. Médias, número de respostas válidas e desvio padrão relativos ao domínio "Apoio"

\begin{tabular}{|c|c|c|c|c|c|c|}
\hline Comando & $\begin{array}{c}\text { Média de } \\
\text { concordância }\end{array}$ & Desvio padrão & $\begin{array}{l}\text { Número de respostas } \\
\text { válidas }\end{array}$ & $\begin{array}{l}\text { Média de } \\
\text { importância }\end{array}$ & Desvio padrão & $\begin{array}{l}\text { Número de } \\
\text { respostas válidas }\end{array}$ \\
\hline $\begin{array}{l}\text { 6. O apoio foi oferecido em tempo } \\
\text { oportuno. }\end{array}$ & 4,70 & 0,59 & 74 & 4,86 & 0,41 & 90 \\
\hline $\begin{array}{l}\text { 7. A minha necessidade de ajuda foi } \\
\text { reconhecida. }\end{array}$ & 4,77 & 0,55 & 66 & 4,86 & 0,55 & 90 \\
\hline $\begin{array}{l}\text { 8. Eu senti-me apoiado pelo professor } \\
\text { durante a simulaçãa. }\end{array}$ & 4,79 & 0,51 & 63 & 4,93 & 0,25 & 90 \\
\hline $\begin{array}{l}\text { 9. Eu fui apoiado no processo de } \\
\text { aprendizagem. }\end{array}$ & 4,89 & 0,36 & 72 & 4,93 & 0,25 & 90 \\
\hline Total & 4,77 & 0,45 & 77 & 4,89 & 0,28 & 90 \\
\hline
\end{tabular}


A tabela 3, corresponde aos resultados da análise das médias e desvio padrão dos itens numerados de 10 a 14 que correspondem a afirmações sobre "Resolução de problemas" da simulação.

Tabela 3. Médias, número de respostas válidas e desvio padrão relativos ao domínio "Resolução de problemas"

\begin{tabular}{|c|c|c|c|c|c|c|}
\hline Comando & Média de concordância & Desvio padrão & $\begin{array}{c}\text { Número de respostas } \\
\text { válidas }\end{array}$ & $\begin{array}{l}\text { Média de } \\
\text { importância }\end{array}$ & Desvio padrão & $\begin{array}{c}\text { Número de respostas } \\
\text { válidas }\end{array}$ \\
\hline $\begin{array}{l}\text { 10. A resolução de problemas } \\
\text { de forma autônoma foi } \\
\text { facilitada. }\end{array}$ & 4,55 & 0,70 & 85 & 4,76 & 0,66 & 90 \\
\hline $\begin{array}{l}\text { 11. Fui incentivado a explorar } \\
\text { todas as possibilidades da } \\
\text { simulação. }\end{array}$ & 4,53 & 0,86 & 78 & 4,80 & 0,62 & 90 \\
\hline $\begin{array}{l}\text { 12. A simulação foi projetada } \\
\text { para o meu nível específico de } \\
\text { conhecimento e habilidades. }\end{array}$ & 4,63 & 0,78 & 88 & 4,89 & 0,32 & 90 \\
\hline $\begin{array}{l}\text { 13. A simulação permitiu-me a } \\
\text { oportunidade de priorizar as } \\
\text { avaliações e condutas } \\
\text { médicas. }\end{array}$ & 4,63 & 0,88 & 89 & 4,83 & 0,50 & 90 \\
\hline $\begin{array}{l}\text { 14. A simulação proporcionou- } \\
\text { me uma oportunidade de } \\
\text { estabelecer objetivos para a } \\
\text { assistência do meu paciente. }\end{array}$ & 4,85 & 0,39 & 88 & 4,94 & 0,23 & 90 \\
\hline Total & 4,65 & 0,51 & 89 & 4,84 & 0,34 & 90 \\
\hline
\end{tabular}


A tabela 4, corresponde aos resultados da análise das médias e desvio padrão dos itens numerados de 15 a 18 que correspondem a afirmações sobre "Feedback/ reflexão" da simulação.

Tabela 4 . Médias, número de respostas válidas e desvio padrão relativos ao domínio "Feedback/Reflexão"

\begin{tabular}{|c|c|c|c|c|c|c|}
\hline Comando & Média de concordância & Desvio padrão & $\begin{array}{l}\text { Número de } \\
\text { respostas válidas }\end{array}$ & $\begin{array}{l}\text { Média de } \\
\text { importância }\end{array}$ & Desvio padrão & $\begin{array}{l}\text { Número de } \\
\text { respostas válidas }\end{array}$ \\
\hline 15. O feedback fornecido foi construtivo. & 4,90 & 0,40 & 90 & 4,98 & 0,15 & 90 \\
\hline $\begin{array}{l}\text { 16. O feedback foi fornecido em tempo } \\
\text { oportuno }\end{array}$ & 4,79 & 0,63 & 90 & 4,87 & 0,43 & 90 \\
\hline $\begin{array}{l}\text { 17. A simulação permitiu-me analisar } \\
\text { meu próprio comportamento e ações. }\end{array}$ & 4,90 & 0,41 & 86 & 4,98 & 0,15 & 90 \\
\hline $\begin{array}{l}\text { 18. Após a simulação houve } \\
\text { oportunidade para obter orientação / } \\
\text { feedback do professor, a fim de } \\
\text { construir conhecimento para outro nível. }\end{array}$ & 4,90 & 0,37 & 90 & 4,97 & 0,24 & 90 \\
\hline Total & 4,87 & 0,31 & 90 & 4,95 & 0,16 & 90 \\
\hline
\end{tabular}


A tabela 5 corresponde aos resultados da análise das médias e desvio padrão dos itens numerados de 19 a 20 que correspondem a afirmações sobre "Realismo" da simulação.

Tabela 5. Médias, número de respostas válidas e desvio padrão relativos ao domínio "Realismo"

\begin{tabular}{l|c|c|c|c|c}
\hline \multicolumn{1}{c|}{ Comando } & $\begin{array}{c}\text { Média de } \\
\text { concordância }\end{array}$ & Desvio padrão & $\begin{array}{c}\text { Número de respostas } \\
\text { válidas }\end{array}$ & $\begin{array}{c}\text { Média de } \\
\text { importância }\end{array}$ & $\begin{array}{c}\text { Desvio padrão } \\
\text { respostas válidas }\end{array}$ \\
\hline $\begin{array}{l}\text { 19. O cenário se assemelhava a uma } \\
\text { situação da vida real. }\end{array}$ & 4,94 & 0,23 & 4,93 & 0,29 \\
$\begin{array}{l}\text { 20. Fatores, situações e variáveis da vida } \\
\text { real foram incorporados ao cenário de } \\
\text { simulação. }\end{array}$ & 4,94 & 0,23 & 90 & 4,93 \\
\hline Total & & 9,94 & 90 & 4,93 \\
\hline
\end{tabular}




\section{Discussão}

A simulação realística tem se difundido na área do ensino médico como uma abordagem educacional na qual o aprendizado acontece através da experiência individual. Então, o aluno, sendo exposto a uma situação de prática clínica, tem a oportunidade de praticar a execução de habilidades teóricas adquiridas previamente, em um ambiente seguro e controlado. O método em questão, está associado ao aprimoramento da construção do conhecimento e desenvolvimento psicomotor, cognitivo e comportamental do aluno, assim como uma maior disposição e confiança no processo de aprendizado. $\underline{\underline{8}, 9}$

Diante do cenário clínico aplicado, com o tema: "abordagem clínica do paciente com demência", os alunos, em sua maioria, avaliaram de forma positiva o design construído para a simulação aplicada assim como a importância da elaboração adequada do design no processo de aprendizagem.

Dentre os domínios analisados, aquele que apresentou maior média de concordância na construção da simulação foi o domínio "Realismo". Esse dado demonstra que a maior parte dos alunos acredita que o cenário se assemelhava, de fato, a uma situação real e que fatores, situações e variáveis da vida real foram devidamente incorporados ao cenário da simulação.

O domínio "feedback/reflexão" obteve média de concordância pouco inferior ao "Realismo", seguido por "Apoio" e "Objetivos e informações", que apresentaram concordâncias equivalentes e intermediárias entre os domínios.

Por fim, o domínio que apresentou pior desempenho na concordância dentro da avaliação dos estudantes foi o "Resolução de problemas". Então, ainda que maior parte tenha apresentado concordância, muitos alunos não se sentiram preparados para resolver os problemas levantados durante a simulação de forma autônoma, apresentando dificuldades que podem ter sido decorrentes do nível da situação problema em questão, pela falta de incentivo do professor na resolução e pela inabilidade do aluno em definir condutas e objetivos no atendimento.

Na análise das médias de importância, o domínio com melhor desempenho, por consequência classificado como mais importante, foi o "Feedback/reflexão", seguido por "Realismo", "Objetivos e informações", "Apoio" e "Resolução de problemas".

Os resultados encontrados neste estudo são equivalentes ao estudo "Percepções de estudantes de enfermagem acerca das dimensões estruturais da simulação clínica", publicado em 2019 pela Scientia Medica, que também utiliza a Escala de Design da Simulação para descrever a estrutura de um cenário de simulação aplicado em estudantes de Enfermagem. ${ }^{\mathbb{I}}$ Isso quer dizer que ambos os estudos apresentaram resultados com predominância positiva de concordância em todos os domínios de avaliação da escala, assim como importância também predominantemente positiva de todos esses aspectos. Apesar disso, os estudos se diferem nos aspectos de melhor desempenho (maior concordância) avaliado pelo aluno quando comparados os domínios entre si, fato que pode decorrer da construção distinta do cenário, tema, prática e orientador no cenário da simulação.

Basak et al. $\frac{10}{}$ também utilizou a Escala de Design da Simulação para estabelecer um comparativo entre simulações aplicadas a alunos também do curso de Enfermagem que utilizavam manequins de alta e baixa fidelidade. Um dos resultados desse estudo demonstrou que para os grupos de estudantes avaliados, a simulação realística que utilizou manequins de alta fidelidade atingiu escores maiores no domínio "Realismo", quando comparado a simulação com manequim de baixa fidelidade. Esse resultado entra em concordância com o presente estudo, demonstrando o benefício que se pode ter em utilizar a escala como guia para a construção, desenvolvimento e aperfeiçoamento do design da simulação, pensando em obter um maior aproveitamento por parte dos alunos. Além disso, o estudo de Basak et al. foi capaz de associar melhores resultados de satisfação do aluno e autoconfiança ao cenário que utilizou manequins de alta fidelidade, que foi, por consequência, o mais realístico. ${ }^{10}$ Essa hipótese é condizente com a avaliação dos alunos deste estudo, considerando que aqui foram utilizados atores como pacientes simulados, que classificam o domínio "Realismo" como o segundo mais importante dentre os domínios avaliados.

Outro estudo realizado por Reese et al. encontrou resultados semelhantes com o domínio "Feedback/ Reflexão" como o mais importante e de maior concordância, assim como neste estudo, que trouxe o mesmo domínio como o melhor classificado em concordância e importância.11 
O presente estudo limita-se pela não validação da escala no grupo participante em outros estudos prévios.

\section{Conclusão}

Os resultados obtidos através desse estudo são capazes de indicar percepções predominantemente positivas dos alunos do curso de medicina acerca do design construído para a simulação aplicada, bem como o reconhecimento de sua importância no processo de aprendizagem.

\section{Contribuições das autoras}

Todas as autoras contribuíram equilibradamente para a concepção e relato do estudo.

\section{Conflitos de interesses}

Nenhum conflito financeiro, legal ou político envolvendo terceiros (governo, empresas e fundações privadas, etc.) foi declarado para nenhum aspecto do trabalho submetido (incluindo, mas não limitando-se, a subvenções e financiamentos, participação em conselho consultivo, desenho de estudo, preparação de manuscrito, análise estatística, etc.).

\section{Referências}

1. Villagrán I, Tejos R, Chahuan J. Percepción de estudiantes de pregrado de Medicina de talleres de simulación de procedimientos médico-quirúrgicos. Rev. Med Chile [Internet]. 2018;146(6). Disponível em: https://scielo.conicyt.cl/pdf/rmc/ v146n6/0034-9887-rmc-146-06-0786.pdf

2. Meakim C, Boese T, Decker S, Franklin AE, Gloe D, Lioce Lori, et al. Standards of Best Practice: Simulation Standard I: Terminology. Clin. Simul. Nurs. 2013;9(6):S3-11. http://dx.doi.org/10.1016/j. ecns.2013.04.001
3. Brandão CFS, Collares CF, Marin HF. A simulação realística como ferramenta educacional para estudantes de medicina. Sci med [Internet]. 2014;24(2):187-92. Disponível em: https:// revistaseletronicas.pucrs.br/ojs/index.php/scientiamedica/article/ view/16189/11485

4. Parolin I. As emoções como mediadoras da aprendizagem. VII Encontro de Educação da PUCPR - EDUCERE Saberes Docentes; 2007 nov. 5-8; Curitiba, PR.

5. Flato UAP, Guimarães HP. Educação baseada em simulação em medicina de urgência e emergência: a arte imita a vida. Rev Bras Clin Med. 2011;9(5):360-4. Disponível em: http://files.bvs.br/ upload/S/1679-1010/2011/v9n5/a2250.pdf

6. Almeida RGS, Mazzo A, Martins JCA, Pedersoli CE, Fumincelli L, Mendes IAC. Validação para a língua portuguesa da Simulation Design Scale. Texto Contexto - enferm. 2015;24(4):934-40. https:// doi.org/10.1590/0104-0707201500004570014

7. Costa RRO, Medeiros SM, Martins JCA, Coutinho VRD. Percepções de estudantes de enfermagem acerca das dimensões estruturais da simulação clínica. Scientia Medica. 2019;29(1):e32972. https://doi.org/10.15448/1980$\underline{6108.2019 .1 .32972}$

8. Al-Ghareeba A, McKennab L, Cooper S. The influence of anxiety on student nurse performance in a simulated clinical setting: $A$ mixed methods design. Int J Nurs Stud. 2019;98:57-66. https://doi. org/10.1016/j.ijnurstu.2019.06.006

9. Coppola A, Coppard B. Impact of Participation in an Interprofessional Acute Care High-Fidelity Simulation for Occupational and Physical Therapy Graduate Students. J Allied Health. 2019;48(4):248-56. Citado em: PMID: 31800654

10. Basak T, Unver V, Moss J, Watts P, Gaioso V. Beginning and advanced students' perceptions of the use of low- and highfidelity mannequins in nursing simulation. Nurse Educ Today. 2016;36:37-43. https://doi.org/10.1016/j.nedt.2015.07.020

11. Reese C, Jefries P, Engum S. Using Simulations to Develop Nursing and Medical Student Collaboration. Nurs Educ Perspect. 2010;31(1):33-7. Citado em: PMID: 20397478 
Anexo A. Escala de Design da Simulação

BBSIIANA

Nome:

Cenário:

data:

/__

1. ESCALA DE DESIGN DA SIMULAÇĀO (Simulation Design Scale Student Version-National League for Nursing, 2013)). A fim de avaliar se os melhores elementos do plano de simulação foram implementados, responda ao questionário abaixo de acordo com a sua percepção. Nāo existem respostas certas ou erradas, apenas o seu nivel de concordância ou discordância. Por favor, use o código a seguir para responder as perguntas.

\begin{tabular}{|c|c|c|c|c|c|c|c|c|c|c|c|c|}
\hline \multicolumn{8}{|c|}{$\begin{array}{l}\text { Use o seguinte sistema de classificaçāo para avaliar as práticas educativas: } \\
1 \text { - Discordo totalmente da afirmaçāo } \\
2 \text { - Discordo da afirmaçāo } \\
3 \text { - Indeciso - nem concordo nem discordo da afirmaçāo } \\
4 \text { - Concordo com a afirmaçāo. } \\
5 \text { - Concordo totalmente com a afirmaçāo } \\
\text { NA - Näo aplicável, a declaraçāo nāo diz respeito à atividade simulada realizada }\end{array}$} & \multicolumn{5}{|c|}{$\begin{array}{l}\text { Avalie cada item com base em } \\
\text { quāo importante este é para vocé. } \\
\text { 1-Näo é importante } \\
\text { 2-Um pouco importante } \\
\text { 3-Neutro } \\
\text { 4-Importante } \\
\text { 5- Muito Importante }\end{array}$} \\
\hline \multicolumn{2}{|c|}{ COMANDOS } & 1 & 2 & 3 & 4 & 5 & NA & 1 & 2 & 3 & 4 & 5 \\
\hline \multirow[t]{5}{*}{$\begin{array}{l}\text { Objetivose } \\
\text { informaçōes }\end{array}$} & $\begin{array}{l}\text { 1. No inicio da simulação foi fornecida } \\
\text { informaçāo suficiente para proporcionar } \\
\text { orientaçäo e incentivo. }\end{array}$ & 1 & 2 & 3 & 4 & 5 & NA & 1 & 2 & 3 & 4 & 5 \\
\hline & $\begin{array}{l}\text { 2. Eu entendi claramente a finalidade e os } \\
\text { objetivos da simulação. }\end{array}$ & 1 & 2 & 3 & 4 & 5 & NA & 1 & 2 & 3 & 4 & 5 \\
\hline & $\begin{array}{l}\text { 3. A simulaçāo forneceu informação suficiente, } \\
\text { de forma clara, para eu resolver a situaçāo- } \\
\text { problema. }\end{array}$ & 1 & 2 & 3 & 4 & 5 & NA & 1 & 2 & 3 & 4 & 5 \\
\hline & $\begin{array}{l}\text { 4. Foi-me fornecida informação suficiente } \\
\text { durante a simulaçāo. }\end{array}$ & 1 & 2 & 3 & 4 & 5 & NA & 1 & 2 & 3 & 4 & 5 \\
\hline & $\begin{array}{l}\text { 5. As pistas foram adequadas e direcionadas } \\
\text { para promover a minha compreensāo. }\end{array}$ & 1 & 2 & 3 & 4 & 5 & NA & 1 & 2 & 3 & 4 & 5 \\
\hline \multirow[t]{4}{*}{ Apoio } & 6. 0 apoio foi oferecido em tempo oportuno. & 1 & 2 & 3 & 4 & 5 & NA & 1 & 2 & 3 & 4 & 5 \\
\hline & $\begin{array}{l}\text { 7. A minha necessidade de ajuda foi } \\
\text { reconhecida. }\end{array}$ & 1 & 2 & 3 & 4 & 5 & NA & 1 & 2 & 3 & 4 & 5 \\
\hline & $\begin{array}{l}\text { 8. Eu senti-me apoiado pelo professor durante } \\
\text { a șimulação. }\end{array}$ & 1 & 2 & 3 & 4 & 5 & NA & 1 & 2 & 3 & 4 & 5 \\
\hline & $\begin{array}{l}\text { 9. Eu fui apoiado no processo de } \\
\text { aprendizagem. }\end{array}$ & 1 & 2 & 3 & 4 & 5 & NA & 1 & 2 & 3 & 4 & 5 \\
\hline \multirow{5}{*}{$\begin{array}{l}\text { Resolução } \\
\text { de } \\
\text { problemas }\end{array}$} & $\begin{array}{l}\text { 10. A resolução de problemas de forma } \\
\text { autônoma foi facilitada. }\end{array}$ & 1 & 2 & 3 & 4 & 5 & NA & 1 & 2 & 3 & 4 & 5 \\
\hline & $\begin{array}{l}\text { 11. Fui incentivado a explorar todas as } \\
\text { possibilidades da simulaçäo. }\end{array}$ & 1 & 2 & 3 & 4 & 5 & NA & 1 & 2 & 3 & 4 & 5 \\
\hline & $\begin{array}{l}\text { 12. A simulação foi projetada para o meu nivel } \\
\text { especifico de conhecimento e habilidades. }\end{array}$ & 1 & 2 & 3 & 4 & 5 & NA & 1 & 2 & 3 & 4 & 5 \\
\hline & $\begin{array}{l}\text { 13. A simulaçāo permitiu-me a oportunidade } \\
\text { de priorizar as avaliaçōes e condutas médicas. }\end{array}$ & 1 & 2 & 3 & 4 & 5 & NA & 1 & 2 & 3 & 4 & 5 \\
\hline & $\begin{array}{l}\text { 14. A simulação proporcionou-me uma } \\
\text { oportunidade de estabelecer objetivos para a } \\
\text { assistềncia do meu paciente. }\end{array}$ & 1 & 2 & 3 & 4 & 5 & NA & 1 & 2 & 3 & 4 & 5 \\
\hline \multirow{4}{*}{$\begin{array}{l}\text { Feedback/ } \\
\text { Reflexāo }\end{array}$} & 15. O feedback fornecido foi construtivo. & 1 & 2 & 3 & 4 & 5 & NA & 1 & 2 & 3 & 4 & 5 \\
\hline & $\begin{array}{l}\text { 16. O feedback foi fornecido em tempo } \\
\text { oportuno }\end{array}$ & 1 & 2 & 3 & 4 & 5 & NA & 1 & 2 & 3 & 4 & 5 \\
\hline & $\begin{array}{l}\text { 17. A simulação permitiu-me analisar meu } \\
\text { próprio comportamento e açōes. }\end{array}$ & 1 & 2 & 3 & 4 & 5 & NA & 1 & 2 & 3 & 4 & 5 \\
\hline & $\begin{array}{l}\text { 18. Após a simulação houve oportunidade para } \\
\text { obter orientaçāo / feedback do professor, a } \\
\text { fim de construir conhecimento para outro } \\
\text { nivel. }\end{array}$ & 1 & 2 & 3 & 4 & 5 & NA & 1 & 2 & 3 & 4 & 5 \\
\hline \multirow[t]{2}{*}{ Realismo } & $\begin{array}{l}\text { 19. O cenário se assemelhava a uma situação } \\
\text { da vida real. }\end{array}$ & 1 & 2 & 3 & 4 & 5 & NA & 1 & 2 & 3 & 4 & 5 \\
\hline & $\begin{array}{l}\text { 20. Fatores, situaçōes e variáveis da vida real } \\
\text { foram incorporados ao cenário de simulaçāa. }\end{array}$ & 1 & 2 & 3 & 4 & 5 & NA & 1 & 2 & 3 & 4 & 5 \\
\hline
\end{tabular}

Referência: Almeida RGS, Mazzo A, Martins JCA, Pedersoli CE, Fumincelli L, Mendes IAC. Validação para a lingua portuguesa da

Simulation Design Scale. Texto Contexto Enferm, Florianópolis, 2015 Out-Dez; 24(4): 934-40. 